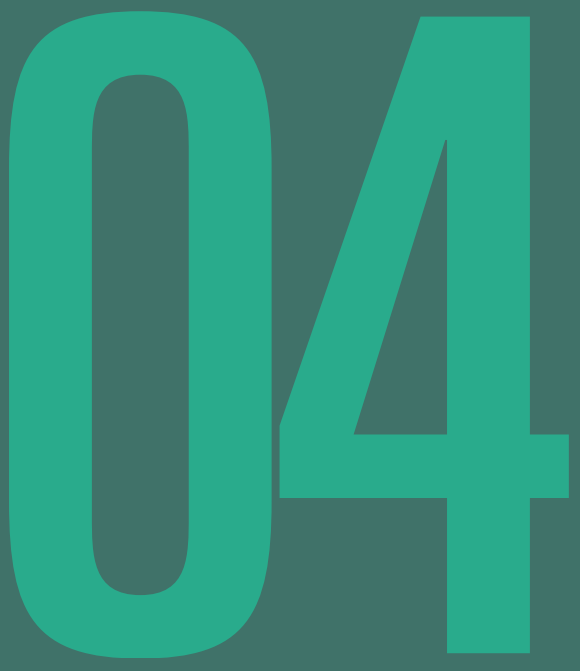

\title{
IMPLEMENTACIÓN DE ESTRATEGIAS DE LECTURA A UN LECTOR
}

\section{Implementation of reading strategies for a reader.}

Dra. Martha Judith Soto Flores https://orcid.org/0000-0003-2615-879X

TP264@correo.tij.ibero.mx Doctora en Artes por la Universidad de Guanajuato Docente en Universidad Iberoamericana Ciudad de México - Tijuana 
Palabras clave: Arte, estrategia, estrategia de lectura, lectura

Keyword: Art, strategy, reading strategy, reading.

Resumen:

El presente trabajo pretende mostrar al lector-académico que -al implementar una estrategia de lectura en sus proyectos de investigación para el área - se puede mejorar y optimizar el resultado de sus textos académicos $y$, al respecto, se plantea que es posible mejorar sus proyectos de investigación al utilizar una estrategia de lectura adecuada a su estudio. Este estudio es una aproximación a la implementación de una estrategia de lectura aplicada al trabajo de un académico-investigador del área de diseño así como otras, donde podemos visualizar un mejoramiento $y$, sobre todo, optimizar tiempos en proyectos de investigación. Al finalizar la investigación se llegó a la conclusión de que -al implementar una estrategia adecuada para cada estudio a realizar por un académico - se puede mejorar el resultado de proyectos de investigación.
Abstract:

This work attempts to show to the academic-reader that -as they implement a reading strategy for their research projects- the result of their academic texts can improve and optimize by implementing an adequate reading strategy for their studies. This study is an approximation to implementing a reading strategy applied to the work of an academic-researcher of the design area as well as other where we can visualize an improvement and above all optimize time in research projects. At the end of the experiment the conclusion was reached that implementing an adequate reading strategy for each study to be conducted by an academic, the result of study projects can be improved for research work. 
Martha Judith Soto Flores

\section{INTRODUCCIÓN}

\section{CONTEXTO GENERAL DE APLICACIÓN}

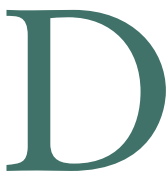

entro de la estructura de la asignatura de Estrategias de Lectura, se contemplan las diversas aproximaciones a la lectura y la forma en que se correlacionan con los diversos objetivos que puede tener el lector en cada caso. De esta manera identificamos la posibilidad de que las estrategias de lectura efectivamente empleadas por un sujeto para un fin determinado sean, o no, las óptimas.

De esta suerte se desprende la oportunidad de elaborar estudios de caso, con el fin de aplicar los conocimientos teóricos adquiridos en esta asignatura para ayudar a lectores específicos a optimizar sus estrategias de lectura para fines determinados. El presente trabajo constituye, precisamente, una primera aproximación a esta aplicación práctica de la asignatura. El caso elegido es el de un académico que realiza trabajo de investigación dentro de su desempeño laboral y se ve, por tanto, en la necesidad de utilizar por lo menos una parte de sus lecturas para obtener información directamente relacionada y útil para su investigación, dentro de marcos de tiempo limitados. Esto hace clara su 
necesidad de utilizar estrategias de lectura que optimicen los resultados que obtiene de su lectura para un fin específico, convirtiéndolo en un caso adecuado para los propósitos que aquí nos ocupan.

\section{IMPORTANCIA DEL PROYECTO}

Determinar las acciones necesarias para optimizar las estrategias de lectura de nuestro sujeto (caso) de estudio resulta ilustrativo y aun típico. Esto tendrá un beneficio tanto para el sujeto como para otros en situaciones similares en la organización del tiempo destinado a la lectura y en la recopilación de datos obtenidos de estas; evitando caer en errores como enfocarse en los detalles de cada tema y perder de vista la relación entre ellos.

\section{OBJETIVOS ESPECÍFICOS:}

- Determinar las estrategias de lectura óptimas de acuerdo con el estado del conocimiento en este campo.

- Determinar las estrategias de lectura efectivamente utilizadas por el sujeto.

- Determinar la coincidencia o falta de coincidencia entre los resultados de los dos objetivos anteriores.

- Elaborar, en su caso, las recomendaciones que correspondan.

\section{CUERPO DEL PROYECTO}

De acuerdo con el método, el primer paso en nuestro estudio consiste en identificar adecuadamente aquello que deseamos estudiar. Tratándose de estudios de caso, como el presente, dicha identificación se realiza con base en tres aspectos principales: el objeto o tema de estudio, el sujeto o caso de estudio y el problema de estudio.

\section{POBLACIÓN-META}

El sujeto de estudio o caso que servirá de base al presente trabajo será el de un académico del área artística que tiene la necesidad de leer para elaborar trabajos de investigación, a saber: 
El Doctor Jaime M. Jiménez Cuanalo, hijo de abogados, tiene 50 años y su aproximación a la lectura fue a la edad de 5 años. Influenciado por sus padres, ha tocado temas como la literatura clásica, la historia, ciencias, la filosofía y el arte. Las lecturas que habitualmente consulta son de corte científico, filosófico y, en menor medida, literario.

El perfil profesional de este académico se integra con estudios de Licenciatura en Derecho y Artes Visuales de la UNAM y la ESAV; así como la maestría en Docencia UABC, obteniendo el grado de Doctor en Artes en la Universidad de Guanajuato. En el 2008 publica su primer libro sobre arte "Arsología una ciencia del arte", posteriormente "Curso de Semiología para artistas y diseñadores” (2017) y su más reciente libro “Tratado General de Semiología” (2019) publicados por la editorial Zona Límite. Ha publicado artículos en diversas revistas y actualmente escribe de forma bimestral para la revista "Zona límite" de publicación electrónica e impresa.

\subsection{ESTRATEGIAS DE LECTURA}

El problema de estudio en este trabajo consiste en determinar si las estrategias de lectura que actualmente posee y practica nuestro sujeto son las óptimas; o si, en su caso, es posible recomendarle otras estrategias de lectura que optimicen su proceso para los fines a que está encaminado.

Primeramente, se le sugiere al lector clasificar sus lecturas en temas; enumerar las lecturas que son más importantes para sus trabajos académicos, las lecturas recreativas que le ayudarán a mejorar su velocidad en la lectura y descansar de los temas que le requieren mayor atención, así como distribuir dichas lecturas en tiempos razonables combinándolo con sus actividades diarias.

Las lecturas recreativas son textos más superficiales y de fácil comprensión, ya que utilizan lenguaje convencional y descriptivo, lo que ayuda a generar lecturas rápidas. Se recomienda que la lectura sea rápida con el fin de analizar el texto en forma global, lo que ayuda al lector a tomar pocos elementos que le facilitarán generar ideas más globales. 
Para las obras de corte académico —que son las referentes a ciencia y filosofía- se recomienda generar lecturas en diferentes fases (Serafini, 2008). La primera que se sugirió fue la fase I: Prelectura, esta le ayudará a evaluar el contenido del texto a leer. En la fase II: lectura crítica del texto, se recomendó leerlo usando subrayado acompañado de palabras clave, lo que al lector le ayudará a mejorar su comprensión de lectura; se trata de generar apuntes en que anotará alguna frase que lo lleve a relacionar lo subrayado con el tema de su investigación. Y, por último, la fase III: poslectura; esta lectura se realiza con la intención de capturar las frases subrayadas y organizarlas en tablas, para que el investigador las tenga a la mano y las pueda consultar.

\subsection{DESCRIPCIÓN ESPECÍFICA DE LA ETRATEGIAS DE LEC- TURA}

Las estrategias de lectura que se sugieren al lector experto fueron desarrolladas de la siguiente manera:

1. Prelectura: Una revisión rápida de textos potencialmente de su interés, en la cual se detectan palabras o temas claves en portada, contraportada, cubiertas interiores o índice.

2. Lectura Rápida: Lectura de corrido del texto completo, para tener una noción general de su contenido global que nos prepare para la siguiente fase.

3. Lectura Crítica: En la cual se sugiere tener una lista de preguntas o cuestiones cuya solución se considera que puede encontrarse dentro del texto antes leído. Aquí se sugiere utilizar el subrayado de aquellas partes claves que conectan con las respuestas buscadas de manera más directa o significativa.

4. Poslectura: Donde organizamos el material obtenido, capturando las citas textuales subrayadas, acotadas por una síntesis del contexto de su extracción, así como de la aportación que, se considera, hacen al tema en cuestión. 
Una vez trazado este plan, el sujeto dio inicio a su práctica eligiendo como primer texto para aplicar esta estructura el titulado "Los 12 Principios del Cerebro", del científico norteamericano especializado en ciencias cognitivas John Medina. A continuación, asentamos parte de la bitácora en la que el sujeto registró este proceso:

\section{PRELECTURA:}

En una visita a la librería, consultando la sección de ciencia y divulgación científica detectó este libro. Lo primero que le llamó la atención fue el dibujo de la portada que es un esquema de la silueta de una cabeza humana donde se ven engranajes en el interior, inmediatamente leyó el título: “que no me gustó demasiado, Los 12 Principios del Cerebro” (Medina, 2010), porque le sonó como a 'Self-Help' o algo así. Pero luego leyó el subtítulo: “Una explicación sencilla de cómo funciona para obtener el máximo desempeño” y eso le dio curiosidad.

Para determinar si se trataba de charlatanería o de un libro auténticamente científico — pero dirigido al público general— pidió que le abrieran el libro y leyó las solapas interiores. Encontró rápidamente la reseña biográfica del autor y su formación científica le pareció sólida. Leyó el índice y quedó un tanto indeciso por los títulos de los primeros capítulos, pero algunos a partir del segundo cuarto del índice le parecieron suficientemente interesantes para tomar el riesgo de comprarlo y leerlo.

Aun así, decidió leer íntegramente la introducción que es de sólo 6 páginas, cosa que pudo hacer parado en la tienda, en un tiempo muy corto. En esa primera aproximación encontró un par de párrafos que le convencieron:

En esas travesías, de vez en cuando me topaba con artículos y libros que hacían algunas afirmaciones sorprendentes -basadas en 'adelantos recientes' de la neurociencia -sobre cómo cambiar la manera de enseñar y hacer negocios. Esto me infundía pánico, y me llevaba a preguntarme si esos autores conocían publicaciones de las que yo no tenía conocimiento alguno. Yo que habló distintos dialectos de la neurociencia, [...] Pero no había porque sentir pánico, pues podemos entrenar responsablemente un ojo escéptico ante cualquier afirmación 
de que la investigación sobre el cerebro puede decirnos, sin equívoco alguno, cómo ser mejores profesores, padres, empresarios o estudiantes. Este libro es un llamado a investigar, sencillamente porque no sabemos lo suficiente como para dictar fórmulas, es un intento de vacuna contra mitos como el del 'efecto Mozart' y el de las personalidades del cerebro izquierdo / cerebro derecho, y el de los métodos para lograr que sus hijos entren a la mejor universidad haciéndoles oír grabaciones de distintos idiomas mientras están en el útero. (Medina, 2010, p. 4-5)

Estas cuantas frases, especialmente lo que se han remarcado en negritas, junto con la formación del autor y su desempeño público y notorio en el campo de las neurociencias, le convencieron de comprar y leer este libro.

\section{LECTURA RÁPIDA:}

En la lectura rápida comenzó por analizar la estructura de los capítulos y descubrió que cada capítulo comienza con una portada en la que el título de este sienta una premisa, por ejemplo:

\section{EJERCICIO / PRINCIPIO \#1 / EL EJERCICIO ES- TIMULA LA POTENCIA CEREBRAL.}

"Noté también que, al final de cada capítulo hay un resumen que explica la premisa y expresa sus consecuencias"; por ejemplo: Resumen / Principio \#1 /El ejercicio estimula la potencia cerebral/

- Nuestro cerebro fue creado para caminar... ¡Veinte kilómetros diarios!

- Si quiere mejorar sus capacidades cognitivas, muévase.

- El ejercicio lleva sangre al cerebro, proporcionándole así la glucosa que le da energía y el oxígeno que absorbe los electrones tóxicos desechados. También estimula la proteína que mantiene conectadas a las neuronas.

- Realizar ejercicio aeróbico dos veces por semana disminuye a la mitad el riesgo de padecer demencia general y disminuye el riesgo de padecer Alzheimer en un sesenta por ciento. (Medina, 2010, p. 32) 
A partir de aquí entré en el cuerpo del capítulo y observé que al principio de cada capítulo se usan anécdotas para introducir y ejemplificar el tema por medio de casos opuestos; uno que corresponde al principio enunciado, por ejemplo, el caso de alguien que haciendo ejercicio envejeció con gran claridad mental, y otros que muestran lo contrario, como el caso de alguien que no hacía ejercicio y a la misma edad que el primer caso había perdido buena parte del uso de sus facultades mentales. Posteriormente se da un recuento de la ciencia disponible al respecto y se aclara, de paso, lo que en realidad no sabemos, pero se encuentra en la cultura popular ya sea por una mala interpretación del estado de la ciencia (casi siempre por exageración) o, de plano, por charlatanería.

Finalmente se plantean las posibles aplicaciones prácticas del principio tanto en el ámbito educativo como en el de los negocios. De esta manera, determinó que - para una primera lectura rápida- lo que le convenía era leer las portadas y resúmenes de cada capítulo, complementadas con el último o últimos dos subtítulos de cada uno, para saber rápidamente la propuesta del autor, dejando para después el análisis minucioso de sus datos y argumentos.

\section{LECTURA CRÍTICA:}

Después de la lectura rápida, quedó profundamente impresionado con el trabajo de Medina, pues le resultó automático conectarlo con los temas de pedagogía que había visto en la maestría - hace poco- y con los temas de la Arsología, que son parte de su propia investigación. En este contexto, las proposiciones de Medina parecían tener aún mucho más utilidad y aplicación práctica de lo que él mismo parece creer, pues, si bien el estado de la investigación de neurociencias no permite hacer propuestas del todo concretas, sí parece fundamentar algunos modelos pedagógicos y contradecir otros - en el mismo sentido en que lo hace la investigación en el campo de la Arsología-. De esta manera, tres campos científicos presentan modelos concurrentes, lo cual evidentemente los refuerza y extiende.

Así, resultó de sumo interés entrar en la lectura a profundidad del texto para descubrir: 
- Qué principios de las neurociencias resultan argumentos a favor de determinados modelos pedagógicos.

- Qué principios de las neurociencias resultan argumentos a favor de partes del modelo de la Teoría General del Arte propuesta por la Arsología.

- De qué modo la pedagogía y la Arsología pueden complementar los 12 Principios del Cerebro y, especialmente, aterrizarlos en aplicaciones prácticas para la educación en general y para la educación artística en particular.

Las cuestiones arriba anotadas sirvieron a modo de cuestionario o guía de lectura, a partir de la cual se pude detectar de manera más eficiente las partes del texto que valía la pena registrar como citas textuales y la forma de sintetizar el resto a manera de paráfrasis o comentario en torno a esas citas textuales. A continuación, ejemplifico algunos de los subrayados que realicé en la introducción:

a. [...] nuestra historia evolutiva nos dice lo siguiente: el cerebro parece estar diseñado para resolver problemas relacionados con la supervivencia en entornos inestables, y hacerlo en un movimiento casi constante. A esto lo llamo “alcance de la función del cerebro”. (Medina, 2010, p. 5)

b. La inestabilidad ambiental condujo a la enorme flexibilidad del cableado cerebral que nos permite resolver problemas mediante la exploración. Aprender de nuestros errores de modo que pudiéramos sobrevivir al aire libre implicaba prestar atención a ciertas cosas a costa de otras, e implicaba crear recuerdos de un modo particular. (Medina, 2010, p. 5-6)

c. ¿Qué demuestran estos estudios, visto en conjunto? Principalmente lo siguiente: Si usted quisiera crear un ambiente educativo directamente opuesto a lo que se le da bien al cerebro, probablemente diseñaría algo similar a un salón de clase. Si quisiera crear un ambiente empresarial directamente opuesto a lo que se le da bien al cerebro, probablemente diseñaría algo parecido a un cubículo de oficina. Y si quisiera cambiar esto, probablemente tendría que acabar con ambos y empezar de nuevo. (Medina, 2010, p. 6) 


\section{POSLECTURA:}

En la poslectura, trató de correlacionar lo subrayado con las preguntas guía que había planteado. Por ejemplo, en el caso de la introducción se realizaron solamente los tres subrayados identificados con los incisos a, b y c. Ahora tocaba determinar de qué manera se correlacionan con los temas guía. El resultado fue como sigue:

P: ¿Qué principios de las neurociencias resultan argumentos a favor de partes del modelo de la Teoría General del Arte propuesta por la Arsología?

R: [...] nuestra historia evolutiva nos dice lo siguiente: el cerebro parece estar diseñado para resolver problemas relacionados con la supervivencia en entornos inestables, y hacerlo en un movimiento casi constante. A esto lo llamo “alcance de la función del cerebro”. (Medina, 2010, p. 5)

Esto se correlaciona con la explicación del papel del arte en el origen de la humanidad de acuerdo con la TGA.

Así, nosotros proponemos el fenómeno llamado arte como un proceso intermedio que enlaza o permite transitar de la inteligencia biológica a la racional, a través del procesamiento material (musical, pictórico, poético, etc.) de la información percibida con los sentidos, dando como resultado las estructuras artificiales primarias o primeras (las obras de arte) con las cuales iremos formando el conocimiento convencional. (Jiménez, 2008: 29)

P: ¿Qué principios de las neurociencias resultan argumentos a favor de determinados modelos pedagógicos?

R: La inestabilidad ambiental condujo a la enorme flexibilidad del cableado cerebral que nos permite resolver problemas mediante la exploración. Aprender de nuestros errores de modo que pudiéramos sobrevivir al aire libre implicaba prestar atención a ciertas cosas a costa de otras, e implicaba crear recuerdos de un modo particular. (Medina, 2010, p. 5-6) 
La consideración de la inestabilidad argumenta a favor del constructivismo educativo, mientras que el aprender mediante la exploración se correlaciona con el ABP (Aprendizaje Basado en Proyectos) y el aprender de nuestros errores se correlaciona con el modelo 'Por Competencias'. Finalmente, como se vería más adelante, el modo especial de crear recuerdos es un argumento a favor de la repetición y práctica repetitiva.

P: ¿De qué modo la pedagogía y la Arsología pueden complementar los 12 Principios del Cerebro y, especialmente, aterrizarlos en aplicaciones prácticas para la educación en general y para la educación artística en particular?

R: Si usted quisiera crear un ambiente educativo directamente opuesto a lo que se le da bien al cerebro, probablemente diseñaría algo similar a un salón de clase. Si quisiera crear un ambiente empresarial directamente opuesto a lo que se le da bien al cerebro, probablemente diseñaría algo parecido a un cubículo de oficina. Y si quisiera cambiar esto, probablemente tendría que acabar con ambos y empezar de nuevo. (Medina, 2010, p.6)

Y tanto la pedagogía como la Arsología aportan en este caso posibles soluciones a este 'empezar de nuevo', como, por ejemplo, la escuela peripatética de Platón en la antigua Grecia, donde se aprendía caminando por un jardín más que sentados en filas de mesabancos dentro de un salón de clase. De esta manera, el material leído y subrayado fue organizado para, a continuación, iniciar su captura en la aplicación 'Notes' del programa 'Word' de modo que estuviera organizado y disponible para su posterior utilización.

\section{CONCLUSIONES}

Respecto del problema de estudio abordado en este trabajo, se concluye que el sujeto (lector académico), a pesar de tener práctica en la lectura desde una edad temprana, así como en su educación básica y profesional, no contaba con un método o estrategias de lecturas implementado de manera óptima respecto a sus objetivos de lectura. El lector académico no construyó su relación con la lectura mediante ningún tipo de instrucción formal escolarizada, sino que su acercamiento a la lectura era completamente intuitivo y poco ordenado. 
Después de sugerir al lector las estrategias de lectura se observó que los hábitos de lectura mejoraron su trabajo de investigación. Fue más eficiente el poder controlar los tiempos y organizar sus lecturas conforme al avance obtenido en las horas de trabajo, asimilando el contenido de las lecturas sin la necesidad de relecturas innecesarias.

Después de implementar las recomendaciones diseñadas de manera específica para la optimización de sus procesos de lectura, el sujeto expresó encontrar mejoras en su organización y aprovechamiento de sus tiempos dedicados a esta actividad, obteniendo así un mayor dominio en su trabajo como investigador.

De esta primera aproximación concluimos que es muy viable obtener resultados útiles mediante la aplicación de las estrategias de lectura para optimizar el aprovechamiento del tiempo dedicado a esta actividad; sin embargo, nos parece claro que hacen falta más estudios de casos similares al presente para poder determinar con precisión el grado real y general de utilidad de este proceso, así como los factores demográficos y de otros tipos que influyen en los resultados.

Proponemos la continuación de este tipo de estudios de caso, a fin de poder recopilar suficiente información para descubrir tendencias en estos procesos y así implementar una auténtica 'Tecnología Educativa' que saque el mayor provecho de estas estrategias de lectura que tan buen primer resultado nos han arrojado. 


\section{BIBLIOGRAFÍA}

Cassany, D. (2008). Prácticas Letradas Contemporáneas. México: Ríos de Tinta. Jiménez C. J. (2008). Arsología una ciencia del arte. México: Zona Límite.

Medina, J. (2010). Los 12 principios del cerebro. Colombia: Norma.

Serafini, M. T. (2008). Cómo se estudia. México: Paidós. 